\title{
GUEST EDITORIAL \\ Challenges of the third decade of life: The significance of social and psychological resources
}

\author{
Marlis Buchmann \\ Heike Solga \\ University of Zurich, Switzerland \\ Berlin Social Science Center and Freie Universitaet Berlin, Germany
}

(Received October 2016

Revised November 2016)

http://dx.doi.org/10.14301/Ilcs.v8i1.437

Status transitions to adulthood have been delayed well into the third decade of life in advanced Western societies over recent years. The third decade has thus mutated into a life period in which young people face more key status transitions and life decisions than ever before. It is therefore of particular interest to learn more about how young people cope with these social and developmental transitions. While psychological and social resources can be assumed to play a key role, research has paid little attention to differences in the social, psychological, and physical resources with which young people start the (challenging) third decade of life. Hence, the question of how resource inequalities experienced in the previous two decades of life are related to young people's opportunities, constraints, and agentic capacities for adopting adult roles has barely been addressed.

This special section aims to partially fill this gap and to provide much needed insight into the antecedents and consequences of inequalities in various kinds of resources among 20-somethings of different social groups and across various societal contexts. The resources targeted in the four papers are physical, psychological, and social. The authors assess how they help to successfully deal with the extended period of transitions into adulthood in the third decade of life and how they affect the development of adulthood commitments and wellbeing. The consequences of young adults' exposure to critical contexts for resource acquisition in the transition to adulthood are also scrutinised. It is highly commendable that all contributions assembled in this Special Section address their particular research questions from a life course perspective. Moreover, they consider how contextual characteristics affect the role of resources and base the analyses on superb longitudinal data.

The collection of the four papers assembled in this special section makes a unique contribution to our understanding of how unequal starting conditions in the third decade of life, related to variations in both resources and contexts, affect health, wellbeing, and early adulthood commitments. Across the four studies, an astonishingly broad range of social and psychological resources has been taken into account, demonstrating the ways they are intertwined with the social and developmental tasks to be accomplished in early adulthood. Malti and colleagues show for Switzerland, a country known for its well-developed direct democratic political system, how the social resource of friendship quality developed in the second decade of life is associated with the internalised acceptance of the principle of equality in early adult life, considered to be a core principle in democratic societies. Positive experiences in close friendships are likely to provide opportunities to connect with the needs and perspectives of others, thus stimulating concern for social equality. This paper also highlights the importance of moral resources accrued in the second decade of life (sympathy, in particular) for instilling sensitivity to social inequality and related values of social justice. The internalisation of these beliefs and values is widely recognised as an important developmental milestone in young adulthood.

How life satisfaction acts as an important personal resource for coping with challenges in the transition to young adulthood is aptly demonstrated in Upadyaya and Salmela-Aro's study. The authors examine the intricate 
longitudinal interplay between life satisfaction and study/work engagement over the transition from post-comprehensive education to higher education or work, following young Finns from the age of 17 to 25. While life satisfaction, indicating general wellbeing, predicts young people's study/work engagement across the entire time period under consideration, study/work engagement predicts life satisfaction only after the transition to higher education or work at the beginning of the third decade. This finding squarely reminds us that the significance of psychological resources greatly depends on the social context in which they play out, in this case, the structural context of the life course and life course transitions. Along this line of reasoning, the developmental changes in the ways domain-specific engagement (school/work) and general wellbeing relate to each other might be attributed to changes in the stage-environment fit, which is assumed to be higher after the transition to higher education or work.

In the contribution by Schoon and Lyons-Amos, general life satisfaction is the outcome of interest at the brink of the third decade of life. The research focuses on how structural and agentic resources and the ways in which young people manage education and employment transitions between the ages of 16 and 20 years in England affect their general wellbeing. Like the aforementioned Finnish study, Schoon and Lyon-Amos also show how dimensions of agency, such as goal certainty and school engagement, predict young people's life satisfaction at the beginning of the third decade. This is remarkable given the institutional differences in the educational system between Finland and England. The reported effects are upheld even when controlling for the type of education and employment transitions, identified by sequence and cluster analyses. By contrast, transition experiences fully mediate the significance of social resources (measured by a familial cumulative socioeconomic risk index) on young people's life satisfaction at the brink of the third decade of life. Taken together, these findings underscore the independent influence of young people's agentic capacities on how well they are satisfied with life.

A particularly incisive, non-normative life transition is the experience of incarceration. This might be especially the case when this experience occurs in late adolescence or early adulthood - a time that would normally be devoted to establishing an independent life. Because inmates are kept in a 'total institution,' deprived of conventional social contacts and interactions, and confronted with limited education and work opportunities, this experience is prone to have negative (long-term) consequences on a broad range of life dimensions. Esposito and co-authors have addressed this issue for the United States, a country known for currently having the highest incarceration rate in the world. They focus on health consequences of incarceration in young adulthood as this stage in the life course is a particularly critical period for developing poor health. The great merit of this study is that it successfully parses out the influence the experience of incarceration has on health from the social and economic confounders that are linked to both the criminal justice contact and health. The findings document that young adults' incarceration is an important stressor with immediate consequence for mental and general health.

In sum, these four papers address, from different angles and perspectives, how unequal social resources and individual agency accrued over the first two decades of life, and the exposure to social contexts characterised by disparate opportunities and constraints, shape life course outcomes in early adulthood. Using high quality longitudinal data and applying state-of-the-art analytical strategies, they document the intricate longitudinal interplay between opportunities and agency in the particularly sensitive period of the transition to the third decade of life.

\section{Acknowledgements}

The papers published in this special section were first presented at the symposium Challenges in the Third Decade of Life in the $21^{\text {st }}$ Century: Individual Development and Health, Social Opportunities and Inequalities at Castle Herrenhausen, June 25-28, 2014, funded by the VW Foundation (grant number: 88257) and organised by Heike Solga, Marlis Buchmann, Martin Diewald, Anette E. Fasang, Richard Lerner, and Iris D. Litt. We would like to thank the VW Foundation for their generous support and Jeylan Mortimer, section editor of LLCS, for her support of and confidence in our editorial work. 